\title{
Foreshock density holes in the context of known upstream plasma structures
}

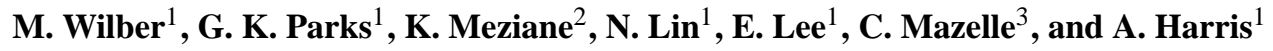 \\ ${ }^{1}$ Space Sciences laboratory, University of California, Berkeley, USA \\ ${ }^{2}$ Department of Physics, University of New Brunswick, Fredericton, Canada \\ ${ }^{3}$ CESR, CNRS/UPS, Toulouse, France
}

Received: 9 May 2008 - Revised: 31 July 2008 - Accepted: 31 July 2008 - Published: 26 November 2008

\begin{abstract}
We present case examples of foreshock density holes and results from a statistical survey, which provide additional characterizations of these recently-described structures. Specific effort is made to place these objects into context with well-studied foreshock phenomena, such as hot flow anomalies (HFAs) and large-amplitude magnetic pulsations (SLAMS). Density holes are observed during higherthan-average solar wind speeds $\left(\sim 620 \mathrm{~km} \mathrm{~s}^{-1}\right)$, have wellcorrelated density and magnetic field intensities, and anticorrelated density and temperature variations. Like HFAs, these structures occur over a wide range of foreshock geometries, suggesting that this is not a determining factor. They are embedded within IMF current sheets, but their crossstructure magnetic shears are considerably lower than for HFAs. When the Cluster spacecraft are widely separated, they are able to measure structure time development, with substantial changes occurring over 10s of seconds, confirming an earlier case study, and possibly indicating short lifetimes as well. We find that density holes can occur in the absence of strong upstream magnetic pulsations and/or density enhancements, which rules out a "wake effect" as the sole explanation for their formation. Most important is the observation that the observed solar wind motional electric fields tend to have components pointing away from the embedding IMF current sheets. Density holes have no connection with magnetic holes and foreshock cavities, and appear not to be early-stage or weakly-formed HFAs.
\end{abstract}

Keywords. Interplanetary physics (Planetary bow shocks) Magnetospheric physics (Plasma waves and instabilities; Solar wind-magnetosphere interactions)

Correspondence to: $\mathrm{M}$. Wilber

(wilber@ssl.berkeley.edu)

\section{Introduction}

Recently, Parks et al. (2006, 2007) reported Cluster and Double Star observations of sub-minute depletions in density and magnetic field intensity upstream of Earth's bow shock. These density holes typically exhibit a greatly reduced solar wind beam that is partially replaced by isotropic suprathermal ions, and often have upstream overshoots in density and field intensity. There is increased wave activity, including enhanced ion cyclotron waves and whistler mode waves in their interiors, along with strong electrostatic (ES) waves near density minima. Although these reports included results from a preliminary survey of events seen during a small number of foreshock transits, no comprehensive characterization has yet been presented. Furthermore, no studies have yet identified specific criteria for when these structures should be observed, nor a mechanism for their production. Notably, those papers indicated that density holes were associated with backstreaming ions characteristic of the foreshock, as well as with moderate cross-structure shears in the magnetic field that indicated they were embedded within interplanetary magnetic field (IMF) current sheets. In addition, strong magnetic pulsations typically have such density holes downstream.

Many shock and foreshock characteristics are strongly determined by geometry, with a critical parameter being $\theta_{B n}$, the angle between the average magnetic field direction and the local shock normal. Quasi-parallel $\left(Q_{\|}\right)$regions have $\theta_{B n} \lesssim 40^{\circ}$ and are characterized by strong, turbulent, ultralow frequency (ULF) waves (Hoppe et al., 1981) and suprathermal, isotropic backstreaming ions that are broad in energy and can extend to $>100 \mathrm{keV}$ (Sentman et al., 1981; Paschmann et al., 1981). Just inside (downstream of) the ULF boundary the foreshock is characterized by waves that instead appear monochromatic, and is populated by backstreaming ions that are observed to be gyrating (peaked at non-zero pitch angles) with energies $<40 \mathrm{keV}$ (Fuselier et al.,

Published by Copernicus Publications on behalf of the European Geosciences Union. 
1986; Meziane et al., 2001). Quasi-perpendicular $\left(Q_{\perp}\right)$ regions $\left(45^{\circ} \lesssim \theta_{B n} \lesssim 70^{\circ}\right)$ typically have very weak wave activity, and coherent, backstreaming field-aligned ion beams (Paschmann et al., 1981; Bonifazi and Moreno, 1981a,b). Strongly perpendicular regions $\left(\theta_{B n} \gtrsim 70^{\circ}\right)$ have much more energetic (100 keV-several MeV) backstreaming gyrating ions (Sarris et al., 1976; Anderson, 1981; Meziane et al., 1999).

Various foreshock plasma structures have been studied over the past three decades, and it is important to place density holes into context. Magnetic pulsations of various forms have been observed since the first spacecraft shock crossings, but of particular interest here are the short, large-amplitude magnetic pulsations (SLAMS) (Greenstadt et al., 1970a,b, 1977; Russell and Greenstadt, 1979; Thomsen et al., 1990; Schwartz and Burgess, 1991) that have been associated with density holes. These are magnetic intensity enhancements at least a factor of $2.5 \times$ the ambient levels, which have time scales on the order of $10 \mathrm{~s}$ in the spacecraft frame, and which are relatively coherent. They are thought to form from steepening foreshock ULF waves (Schwartz, 1991), and to play a critical role in processing the solar wind over a distributed shock transition in $Q_{\|}$regions (Schwartz and Burgess, 1991; Lucek et al., 2008). The orientations of their upstream normals are aligned most closely with upstream field directions, while their maximum interior fields are found at large angles relative to model shock surfaces (Mann et al., 1994). The orientations favor $Q_{\perp}$ geometries for the SLAMS' interior fields, with ion behaviors similar to those at $Q_{\perp}$ shocks. This geometry can lead to some plasma heating.

Lucek et al. (2002, 2004a, 2008) have examined SLAMS using the multi-point capabilities of Cluster and have found that the structures are strongly correlated on spacecraft separation scales of $\sim 100 \mathrm{~km}$, with magnetic field gradient scale sizes $100-150 \mathrm{~km}$, comparable to a solar wind ion inertial length. At larger separations of $\sim 600 \mathrm{~km}$, the structures appear significantly different at each spacecraft. Previously, density depletions had been reported downstream of SLAMS (Thomsen et al., 1990), and were interpreted as "wake effects" due to solar wind flow being partially diverted around the field enhancements (Behlke et al., 2003).

Other well-studied foreshock plasma structures include hot flow anomalies (HFAs) that are characterized by large flow deviations, which at times have sunward-directed $x_{\mathrm{GSE}}$ components, and interior particle temperatures greatly in excess of the ambient solar wind (Schwartz et al., 1985; Schwartz et al., 1988, 2000; Woolliscroft et al., 1986; Thomsen et al., 1986; Thomsen et al., 1988; Thomsen et al., 1993; Paschmann et al., 1988; Lucek et al., 2004b). These appear to be the result of current sheets, specifically, tangential discontinuities (Thomsen et al., 1993), in the IMF interacting with Earth's bow shock. In most cases, the hot interior regions exhibit turbulent plasma and field structure, with field intensities and densities that on average range from a half to several times the exterior solar wind values. (Interior densi- ties tend to remain closer to solar wind values than interior magnetic fields.)

The structure edges are typically enhanced significantly over the ambient solar wind values, and plasma parameters and particle signatures provide evidence that these form fast mode-like shock interfaces with the solar wind (Schwartz et al., 1985; Fuselier et al., 1987; Paschmann et al., 1988; Thomsen et al., 1988). HFAs have magnetosheath manifestations and can impact the magnetopause and magnetosphere (Paschmann et al., 1988; Sibeck et al., 1999; S̆afránková et al., 2002; Eastwood et al., 2008). The paradigm adopted by most authors at this time is that heated interior plasma results in an overpressure relative to the solar wind, with explosive expansion in the solar wind frame that drives the formation of edge shocks (e.g. Thomsen et al., 1986).

As proposed by Burgess (1989), and later confirmed in several observational studies referenced above, as well as in simulations (Thomas et al., 1991), HFAs typically have solar wind convection electric fields with components directed inward on either or both edges of the structures. These motional fields are thought to be important for channeling ions specularly reflected off of the bow shock into the current sheet along its line of connection. Schwartz et al. (2000) have further argued that reflected ions will not successfully reach the current sheet (and thus fill it) unless their gyration velocities exceed the speed at which the current sheet connection line convects along the shock. This condition is best satisfied for IMF discontinuities that have normal orientations with large components transverse to the solar wind flow direction.

Although there is some agreement that the structures described by Thomsen et al. (1986); Fuselier et al. (1987) are of the same class as those considered by, e.g. Schwartz et al. (1985) and Woolliscroft et al. (1986), the former applied somewhat different selection criteria, which is reflected in their original term: hot diamagnetic cavities. Most notably, they considered cases where the magnetic field formed cavities centered on the IMF current sheet, which apparently were the result of the inferred plasma expansion. These hot diamagnetic cavities (HDCs) also exhibited two populations of ions, including a reduced solar wind beam and a backstreaming component. An interpretation is that these represent an early phase of HFA development, with streaming instabilities leading to a single hot interior population later. In a superficial comparison, HDCs resemble the density cavities described by (Parks et al., 2006), but the physical scales for HDCs in the literature are larger, their frequency of occurrence is much lower, and their interior densities and fields do not drop so precipitously.

Entirely different are foreshock cavities (Sibeck et al., 2001, 2002), which are the result of upstream domains with relatively higher interior pressures expanding into adjacent regions. Although these seem similar to HFAs/HDCs, including, e.g. plasma and field compressions on their edges, their interior plasma does not show nearly as strong a temperature increase over neighboring regions and they exhibit no 
strong flow deviations. Rather than resulting from a shockcurrent sheet interaction, these are likely due to pressure differences between adjacent plasma domains. When foreshock regions connected to $Q_{\|}$shocks are embedded within $Q_{\perp}$ regions, the former can be expected to have moderately higher ion pressures. Particles emerging from quasi-parallel shocks are actively scattered by upstream magnetic structures that are convected Earthward, and subsequent interactions with the shock lead to strong particle energization by a Fermi process. In contrast, field-aligned beams apparently emanating from $Q_{\perp}$ shocks are disrupted very slowly, with little apparent in-transit heating (Meziane et al., 2005).

Another class of structures requiring brief mention are solar wind magnetic holes (Turner et al., 1977), which are characterized by significant depletions in the magnetic field intensity, and which exhibit a large range of scales (Stevens and Kasper, 2007). These have been attributed variously to non-propagating current sheets embedded within the solar wind that exhibit diamagnetic pressure balance (Fitzenreiter and Burlaga, 1978; Burlaga and Lemaire, 1978); to the mirror mode instability in the case of small spatial-scale events with small magnetic rotations (Winterhalter et al., 1994); to Alfvénic solitary structures (Baumgärtel, 1999; Buti et al., 2001); and to diamagnetic holes generated through steepened Alfvén waves (Tsurutani et al., 2002) driven by ponderomotive heating of ions. Solar wind magnetic holes seem to be unrelated to density holes, however, on at least two counts: they are not constrained to the ion foreshock, but rather are seen throughout the heliosphere; and they tend to show no changes or increases in associated density (Winterhalter et al., 1994), while a strong density depletion is a defining characteristic for the structures contemplated in this paper. The association of density holes with the ion foreshock suggests that the shock itself or backstreaming ions have a role in their formation.

This study has been motivated by several outstanding questions about density holes: What relationship, if any, do they have to these other well-studied foreshock structures? Density holes are observed in upstream regions that have backstreaming particles and waves characteristic of both quasi-parallel and quasi-perpendicular foreshocks, suggesting that shock geometry is not the determining factor. Could they, for example, be early-stage HFAs, or HFAs that fail to fully develop for some reason? Or can we identify a physical basis for them being fundamentally different? Given that SLAMS generally have density cavities downstream, can the latter be accounted for by the "wake effect" suggested by Behlke et al. (2003)? As we indicate in Sect. 2 following immediately, we have identified a large sample of events (numbering 70) that appear to have no associated large upstream pulsations or density overshoots, for which such a wake effect seems an unlikely explanation. In fact, these density holes are characterized by a greatly diminished reduction in the solar wind beam, and that raises a related question. Absent an upstream pulsation which might divert a fraction of the solar wind, and edge overshoots indicating strong expansion into the surrounding plasma, what mechanism expels the majority of the solar wind beam, or blocks its entry into a structure standing relative to the solar wind flow?

After describing the instrumentation used and criteria for selecting our events in Sect. 2, we begin by presenting a few case events in Sect. 3. These are contrasted with HFAs presented in the literature. We follow in Sect. 4 with results from a statistical survey of carefully-selected events having no strong pulsations upstream, and show that most density hole characteristics are uncorrelated with the magnetic shears across the structures. Further, the normal components of the convection electric field show a statistical tendency to point out of the structures, rather than inward as has been found for HFAs. Finally, after summarizing results we discuss the implications of these results in Sect. 5.

\section{Instrumentation and data selection}

The primary data used for this study are from the $5 \mathrm{eV}-$ $40 \mathrm{keV}$ Cluster/CIS hot ion analyzers (HIA) (Rème et al., 2001) and the FGM fluxgate magnetometer (Balogh et al., 2001), with additional proxy density measurements in the form of spacecraft electric potentials from the EFW electric fields and waves instrument (Gustafsson et al., 1997). The HIA all-ion electrostatic analyzers return to ground moments of the ion distribution functions at spin-resolution (4s), and full 3-D data averaged over 1-3 spins, depending upon the telemetry mode.

Although all events were observed upstream of the bow shock, we have eliminated events recorded when the CIS instrument was in "solar wind" acquisition mode. For this, the sunward-looking high-geometry factor measurements are obtained with the energy sweep truncated below $\sim 1 \mathrm{keV} \mathrm{q}^{-1}$, in order to minimize saturation fluxes that damage the detector. We have found, however, that the low-geometry factor measurements, which are based upon a fraction of velocity space centered on the solar wind beam, do not capture sufficient portions of the supra-thermal ions, and therefore underestimate the temperature. In magnetosheath mode, all of velocity space is measured by the high geometry-factor side of the analyzer, although high fluxes from the fast-moving cold solar wind beam tend to saturate the measurements. The result is an underestimate of the density by factors as high as $2-3$, and a modest overestimate of the temperature, but velocities should remain rather accurate. Inside density holes, where the fluxes are significantly reduced, the saturation errors should be considerably lower. The most error prone measurements therefore are the ratios of exterior to interior densities, which are underestimated.

The survey results presented below employ calibrated, spin-resolution magnetic field data, while case event analyses include calibrated, full-resolution $(22.5 \mathrm{~Hz})$ magnetic field data, all provide by the FGM team. Spacecraft potential 


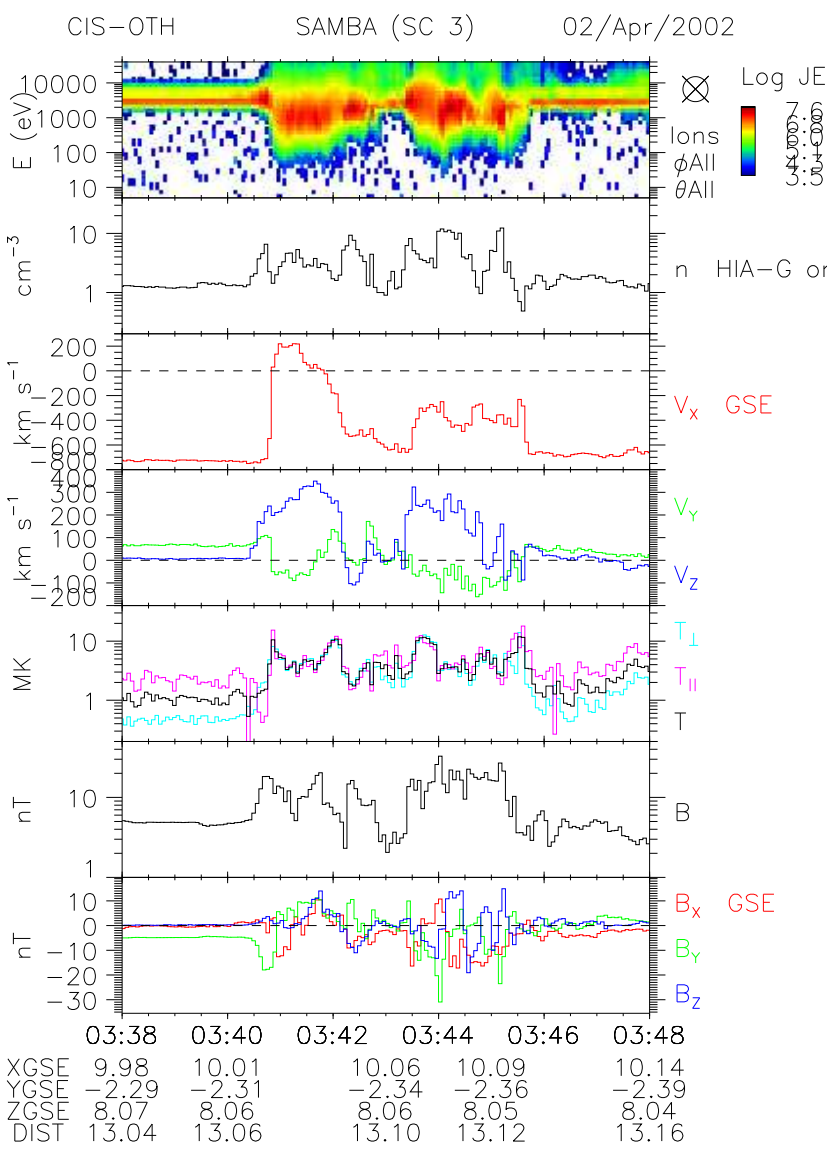

Fig. 1. Hot flow anomaly, 2 April 2002, summary; spin-resolution all-ion spectrogram and moments from CIS/HIA, and magnetic field vectors from FGM (cf. Lucek et al., 2004b).

measurements from EFW serve two important purposes: first, they typically provide proxy density information at $5 \mathrm{~Hz}$ resolution ( $20 \times$ the cadence of the CIS moments); and second, confirm that the sometimes-dramatic density drops observed are not a CIS instrument artifact resulting from aliasing, as might occur were changes to the velocity space location of the cold solar wind beam to cause it to appear antisynchronously with the combined angular and energy sweeps of the analyzer.

In order to disentangle the possible effects of associated plasma structures, we have sought events that lack strong, adjacent upstream pulsations (e.g. SLAMS) or density overshoots. By our criteria, attached fluctuations in spin-averaged density and magnetic field strength must not exceed $2 \times$ the average values upstream (most are much lower), and where strong ULF are present, the nearest upstream maxima must not be significantly different from neighboring maxima where no density holes are present. In other words, adjacent fluctuations must not be distinctive. Of the $\sim 70$ events identified from dates in 2002-2004, almost half were eliminated from the statistical database due to multiple occur- rences or other sub-structure that prevented unambiguous determination of, e.g., density minima or structure widths. Of the events retained, the majority were observed to have suitable characteristics by both spacecrafts 1 (C1) and 3 (C3), for which the CIS/HIA instruments were operational. From the magnetic field and spacecraft potential measurements, we found that almost always the events were of sufficient spatial and temporal scales to be seen by all four probes. In our survey results, we present the $\mathrm{C} 1$ and $\mathrm{C} 3$ measurements separately as a consistency check.

\section{Case events}

\subsection{April 2002 hot flow anomaly}

In order to contrast our observations with those typical of hot flow anomalies, we present in Fig. 1 an example an HFA observed by Cluster and examined in detail by Lucek et al. (2004b). The top panel provides ion energy fluxes from the all-ion HIA instrument, and shows a clear broadening of the spectrum near 03:40:30 UT, which resembles a magnetosheath distribution and includes fluxes extending up to $40 \mathrm{keV}$. In most places, the density for this event is near or in excess of the solar wind values of $1-2 \mathrm{~cm}^{-3}$, although we note a brief gap in the spectrogram and a drop in density at the trailing (upstream) edge near 03:45:30 UT. Very large flow deviations are observed with $v_{x}$ reversing and attaining values $>+200 \mathrm{~km} \mathrm{~s}^{-1}$, and $v_{z}$ reaching $\sim 300 \mathrm{~km} \mathrm{~s}^{-1}$. In places the temperature increased by a factor of 10 over the ambient $1 \mathrm{MK}$ level, with the last peak aligned with the density drop at the upstream edge. The magnetic field is highly structured, with intensity variations comparable to, but not strongly-correlated with, the density variations. Briefly near 03:42:15, and for a half-minute interval starting at 03:42:25 UT the magnetic field intensity drops below the ambient solar wind value, although for most times the field strength exceeds it. It is evident from inspection of the field components before 03:40 and after 03:46 UT that there was a large shear in the IMF direction across the structure.

\subsection{April 2002, density hole}

Figure 2 shows a density hole recorded half an hour after the HFA depicted in Fig. 1. This is a $Q_{\perp} \rightarrow Q_{\|}$transition seen under comparable plasma and field conditions, with $v_{s w} \sim 700 \mathrm{~km} \mathrm{~s}^{-1}$. The change in shock geometry is sensible, since differently-oriented field lines on either side of the current sheet can thread the bow shock at very different locations. The factor of 10 increase in temperature on the interior is comparable to that of the HFA, but in contrast the flow deviation is modest. These spin-resolution data show a strong correlation between the density $n$ and the field intensity $B$, supporting the fast magnetosonic character on the scales sampled. 


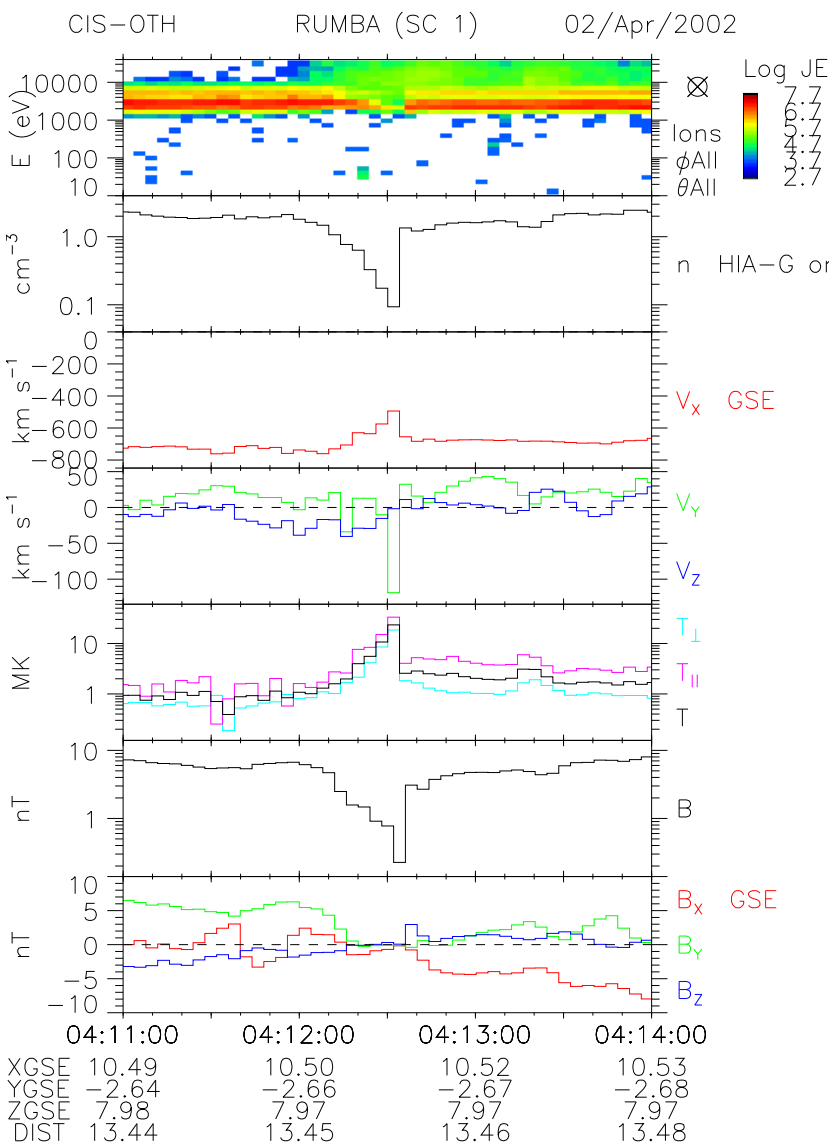

Fig. 2. 2 April 2002 04:11-04:14 UT summary plot. Same format as for Fig. 1.

The $n$ and $B$ profiles, as well as the energy flux spectrogram are intriguing, as the upstream side shows a very abrupt transition, while the downstream side changes gradually. The spectrograms show that on the downstream edge the gradual change occurs with evident energy dispersion, suggesting a possible velocity filter effect. Spectrograms for C3 (not shown) substantiate this dispersion.

Figure 3 shows magnetic field data from FGM and spacecraft potentials, $\phi_{\mathrm{SC}}$, from EFW. Offsets of $1 \mathrm{~V}$ have been applied to the latter to make individual traces easier to discern. A running average of $2 \mathrm{~s}$ has been applied to remove $>1 \mathrm{~Hz}$ structure. Although the maximum in $B$ at 04:12:35 exceeds double the value immediately upstream, we consider the adjacent dip to reflect structure associated with the density hole, rather than the ambient solar wind field intensity.

Putting aside for now the substructure within the middle two dashed vertical lines, there is a clear rotation in the field across the structure. Although $\phi_{\mathrm{SC}}$ varies somewhat crudely with the log of the density, we see that upon entrance into the structure it varies monotonically. The magnetic field traces, on the other hand have additional structure, indicating that on these (kinetic) scales the fast mode-like character of the density hole breaks down.

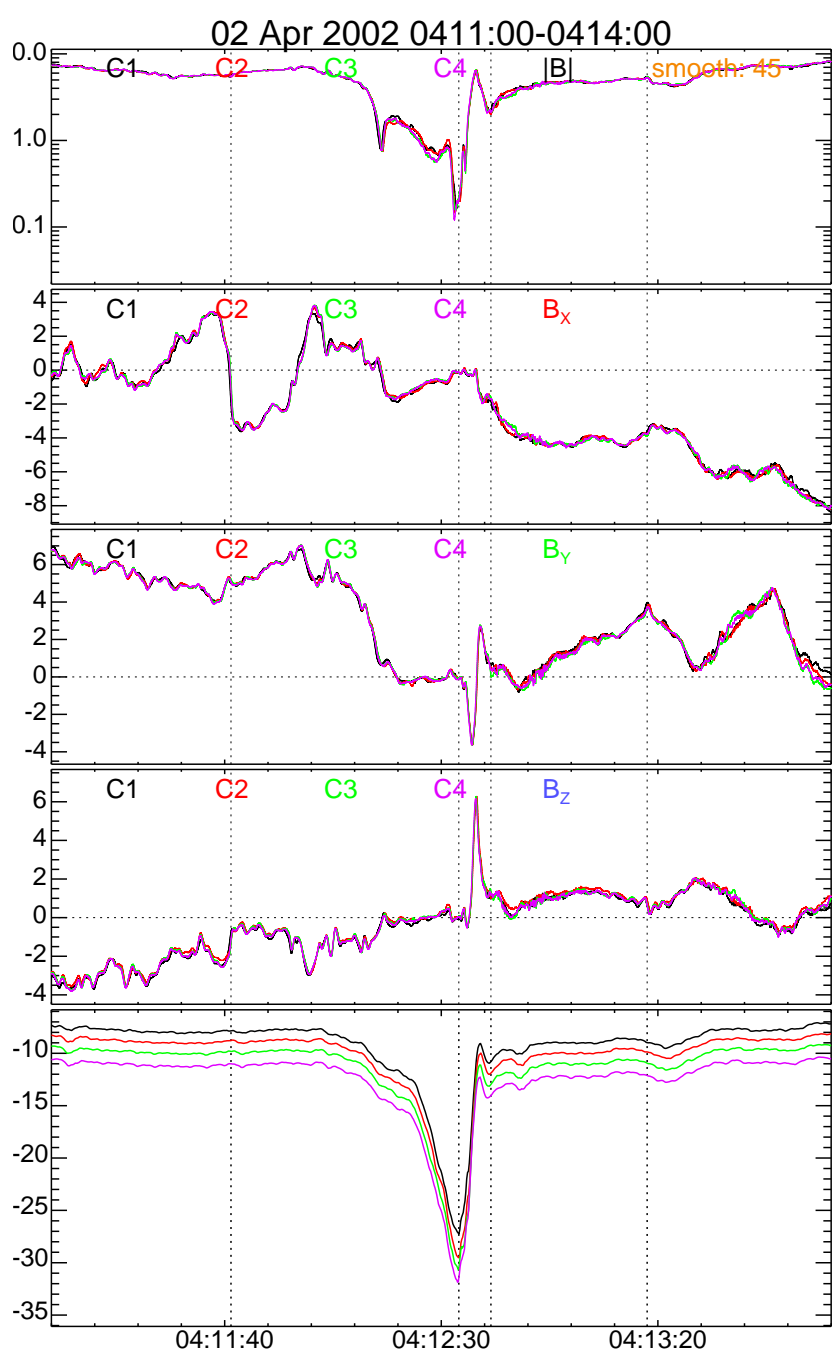

Fig. 3. 2 April 2002 04:11-04:14 UT, magnetic fields $\boldsymbol{B}$ and spacecraft potentials $\phi_{\mathrm{SC}}$; from top down: FGM magnitude (log scale); FGM GSE components; and $\phi_{\mathrm{SC}}$ from EFW. Vertical lines denote intervals for hodograms and timing analysis discussed in text.

In order to test the character of the larger scale rotation in the magnetic field across the structure, we applied a variance analysis to $\boldsymbol{B}$ for the interval delimited by the outer dashed lines in Fig. 3. The normals obtained by the four spacecraft agreed to within $1^{\circ}$, yielding a direction $\boldsymbol{n}_{m v}=[0.30,0.23,0.93]$. Fields from C1 projected onto the variance axes are depicted in the top row of Fig. 4, with the start of the interval in violet and the end in red. This shows a fairly coherent rotation, and no normal component to the minimum variance component, indicating a tangential discontinuity. There is, however, a conspicuous "ear" in green which indicates a momentary deviation of the field from the minimum variance plane. Those points correspond to the brief transition out of the density hole on its upstream edge, which is delimited in Fig. 3 by the interior dashed lines. 

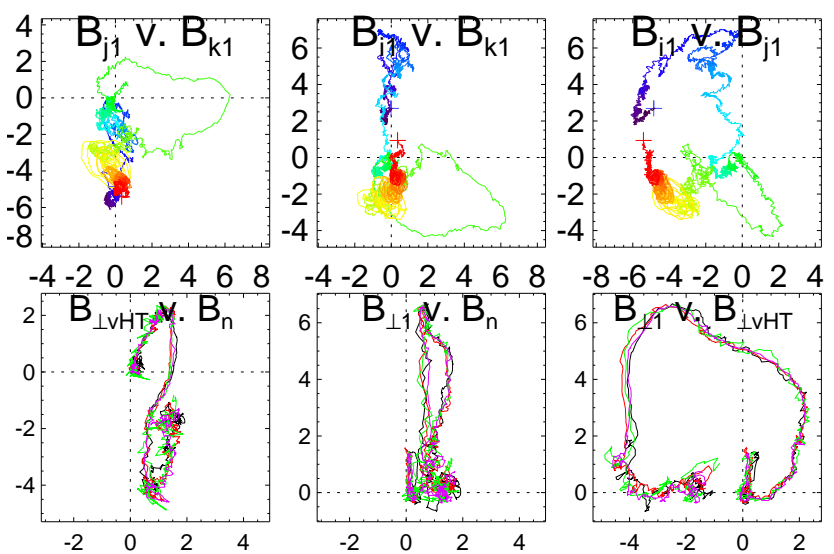

Fig. 4. 2 April $2002 \boldsymbol{B}$ hodograms; top) $\mathrm{C} 1$ in minimum variance coordinates for 04:11:41-04:13:18 UT; bottom) all spacecraft, in timing analysis coordinates for 04:12:34-04:12:41 UT, with, left to right, $\boldsymbol{r}_{H T} \times \boldsymbol{n}_{4 \mathrm{pt}}, \boldsymbol{r}_{\perp} \times \boldsymbol{n}_{4 \mathrm{pt}}, \boldsymbol{r}_{\perp} \times \boldsymbol{r}_{H T}$. See text for details.

We analyzed the density hole's (upstream) exit transition using the multi-point timing analysis method (Harvey, 1998). Although the high solar wind speed of $\sim 700 \mathrm{~km} \mathrm{~s}^{-1}$ and the $\sim 100 \mathrm{~km}$ spacecraft separation scale made this challenging even with FGM's high-resolution data, we were able to obtain sensible, if not accurate, results. The determinations for the downstream edge were inconsistent when using different field components, so we omit them. Application of a variance analysis to the magnetic field over the short exit interval yielded nearly-degenerate medium and maximum eigenvalues, indicating a circularly polarized rotation of the field. We used these two directions to construct maximum and medium variance components for the field, $B_{\max }$ and $B_{\mathrm{med}}$, respectively. These were adopted as two independent components for the timing analysis. Agreement between timing normals from $B_{\max }$ and $B_{\text {med }}$ was to within $5^{\circ}$ of each of the individual minimum variance normals obtained from the four spacecraft, suggesting an excellent direction determination. A third timing normal for the field magnitude $B$ resulted in good agreement with the single-spacecraft normals, here to within $8^{\circ}$.

The average normal direction for the upstream cavity edge obtained from timings of $B_{\max }$ and $B_{\text {med }}$ was $\boldsymbol{n}_{4 \mathrm{pt}}=[0.97,0.24,0.05]$. Normal incidence fast magnetosonic Mach numbers were variable, in part due to small normal flow projection variations, as well as from sampling resolutions that were marginal for this event. We found $M_{A}=1.06 \pm 0.35$, indicating that the upstream edge was standing relative to the solar wind flow at approximately the Alfvén speed.

The bottom row of Fig. 4 shows overlapping hodograms for the magnetic fields of the four spacecraft during the exit transition selection interval. From left to right the panels show projections onto the $\boldsymbol{n}_{4 \mathrm{pt}} \times \boldsymbol{r}_{H T}, \boldsymbol{n}_{4 \mathrm{pt}} \times \boldsymbol{r}_{\perp}$ and $\boldsymbol{r}_{H T} \times \boldsymbol{r}_{\perp}$ planes, where $\boldsymbol{r}_{H T}$ is along the de Hoffman-Teller velocity constructed from the upstream conditions and the normal direction, and $\boldsymbol{r}_{\perp}$ completes an orthogonal coordinate system. There appears to be a small non-zero normal component to the magnetic field $\sim 1 \mathrm{nT}$, and the previously-noted circular polarization is evident in the right panel. Consistent with the small differences between the timing normal and the four minimum variance normals, the four hodograms constructed from minimum variance normals (not shown) are virtually indistinguishable. This suggests that the leading edge of the structure is a rotational discontinuity. For additional examples of magnetohydrodynamic (MHD) discontinuity analyses applied to density holes and associated structures see Lin et al. (2008).

Figure 5 shows a distribution function obtained at 04:12:32 UT, just as the spacecraft was exiting the density hole. The left panel shows a slice through the $v_{\|} \times v_{\perp}$ plane, while the three others show slices in planes perpendicular to $\boldsymbol{B}$ at values of $v_{\|}=0, \pm 800 \mathrm{~km} \mathrm{~s}^{-1}$ relative to the parallel component of the plasma velocity (indicated by dashed colored lines in the left frame). The intensity of the solar wind beam (red contours), included in the $v_{\|} \times v_{\perp}$ slice and in the middle $v_{\perp 0} \times v_{\perp 1}$ slice, is reduced relative to ambient values (not shown). All three slices perpendicular to $\boldsymbol{B}$ show a sharp cut-off near their centers, which is consistent with a remote-sensing distribution obtained at the edge of a sharplydelimited region having occupied guiding centers (Meziane et al., 2003; Schwartz et al., 2006). In contrast, distributions obtained around the downstream edge (not shown) indicate a gradual variation in the supra-thermal population, but no clear remote-sensing edge, consistent with a gradient scale on the downstream edge $>1$ ion Larmor radius.

\subsection{March 2003, nascent density hole}

On 16 March 2003 Cluster observed a nascent density hole forming just upstream of the shock. This occurred during a transition from $Q_{\perp} \rightarrow$ oblique shock geometries. The spacecraft were well separated along $x_{\mathrm{GSE}}$ by $\sim 11200 \mathrm{~km}$, which resulted in a difference of $15 \mathrm{~s}$ between the first and last observation by $\mathrm{C} 3$ and $\mathrm{C} 1$, respectively. Separations transverse to the solar wind flow were only $3800 \mathrm{~km}$, decreasing the likelihood of measuring spatial differences along the structure. Figure 6 shows a summary of plasma parameters for $\mathrm{C} 1$ as it crossed the shock at 22:33:30 UT, and intercepted a small density hole a couple of minutes later at 23:35:15 UT. The spectrogram, which was obtained at 3-spin resolution, does not capture the density hole seen in the 4-s data. Again the solar wind speed was $\sim 700 \mathrm{~km} \mathrm{~s}^{-1}$, with otherwise unremarkable upstream field and plasma values. The density hole was centered on a distinct rotation in the magnetic field, but no density hole is apparent a minute later when the field rotates partially back to its pre-density hole orientation.

Figure 7 shows the magnetic field intensity and the spacecraft potential for a sub-interval 23:33:30-23:37:00 UT, 

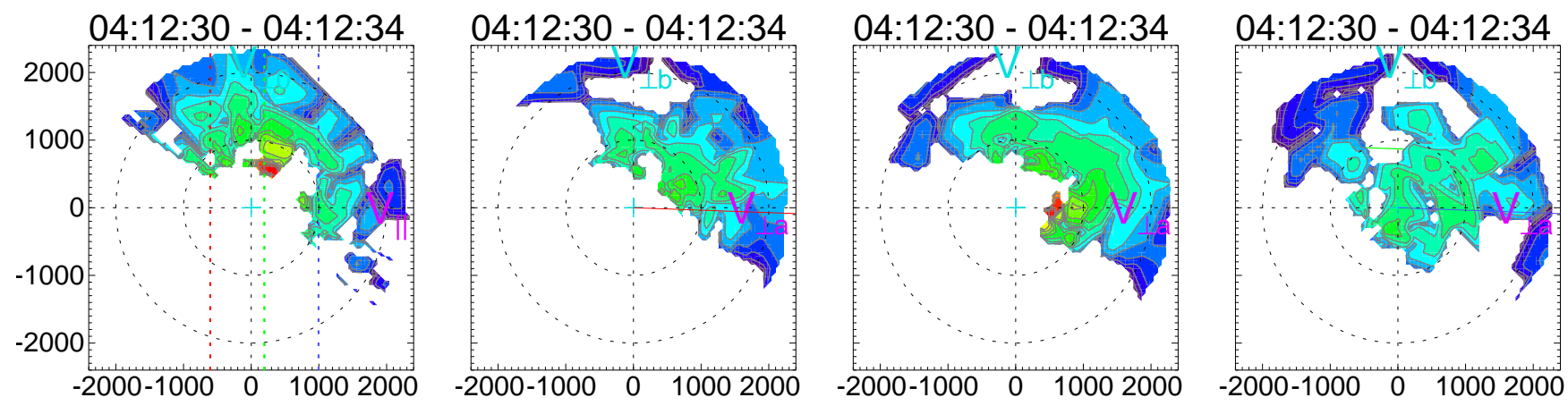

$-29$

$-28$

$-27$

$\log _{10}\left(\mathrm{df} / \mathrm{s}^{3} \mathrm{~cm}^{-6}\right)$

$-25$

$-24$

Fig. 5. 2 April 2002 04:11-04:14 UT, ion distributions from HIA in the spacecraft frame. Left panel shows $v_{\|} \times v_{\perp}$, while the three panels to the right show slices perpendicular to $\boldsymbol{B}$ at values of $v_{\|}=0, \pm 800 \mathrm{~km} \mathrm{~s}^{-1}$ relative to the parallel plasma velocity.

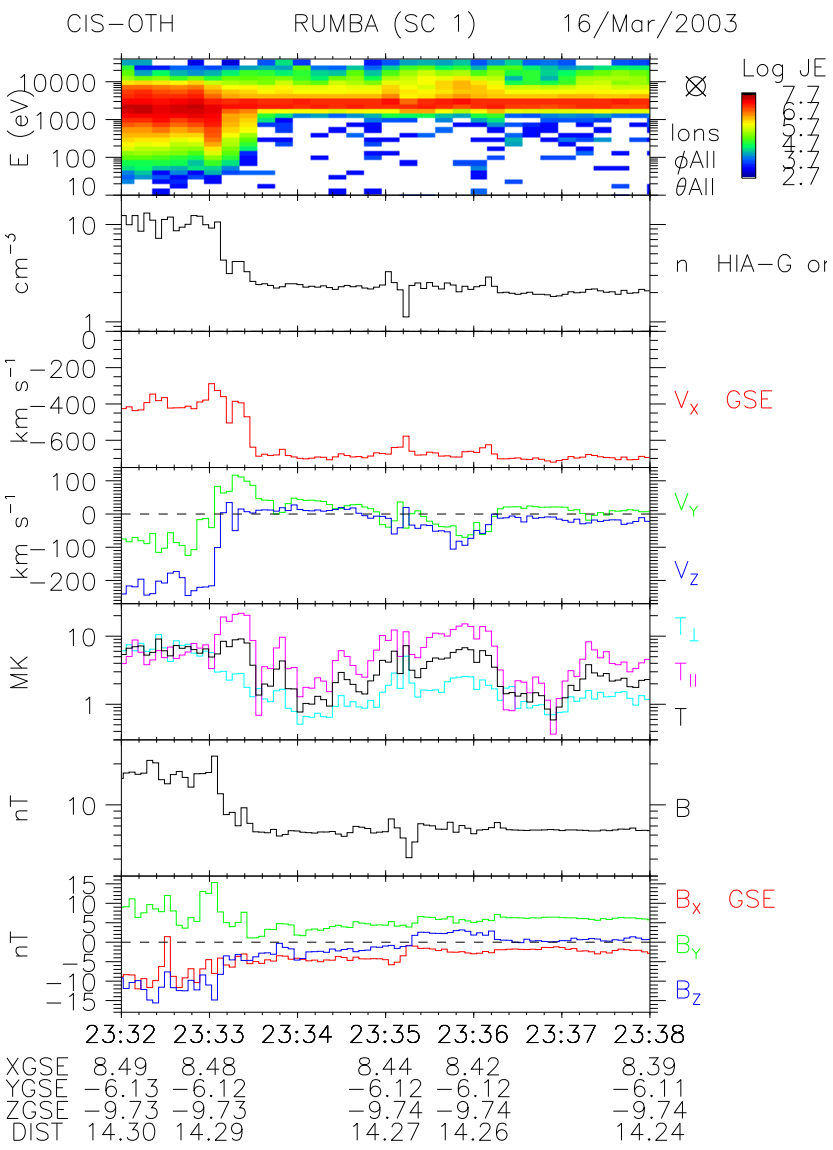

Fig. 6. 16 March 2003 23:32-23:38 UT summary plot. Same format as for Fig. 1.

which excludes the shock crossing observed by $\mathrm{C} 1$. C2, C3 and $\mathrm{C} 4$ detected the density hole at nearly the same time, while $\mathrm{C} 1$ observed it last. $\mathrm{C} 1$ was closest to the shock, where it detected the cavity at its deepest.

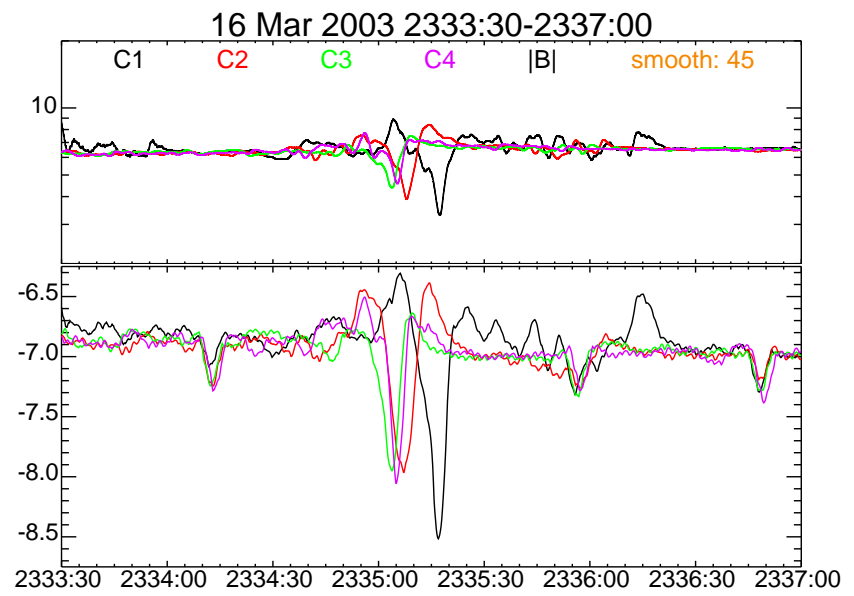

Fig. 7. 16 March 2003 23:33:30-23:37:00 UT, magnetic field intensity and $\phi_{\mathrm{SC}}$.

\subsection{February 2003, time-developing structure}

Not all events observed during the spring of 2003, while Cluster separations were large, show a clear signature of growth. On 19 February the spacecraft recorded a density hole during the passage of an IMF current sheet having a cross-structure magnetic rotation of $\theta_{\Delta B}=141 \pm 8^{\circ}$. We see in Fig. $8 Q_{\perp}$ foreshock characteristics on either side. While there is a very small amount of density overshoot to either side, this is not apparent in the magnetic field data. The solar wind speed was $\sim 550 \mathrm{~km} \mathrm{~s}^{-1}$, and the flow deviation is barely discernible.

Figure 9 shows $B$ and $\phi_{\text {SC }}$ profiles that present somewhat conflicting pictures of what the structure is doing as it passes by the spacecraft formation. The spacecraft potentials suggest a mild broadening of the structure, with accompanying 


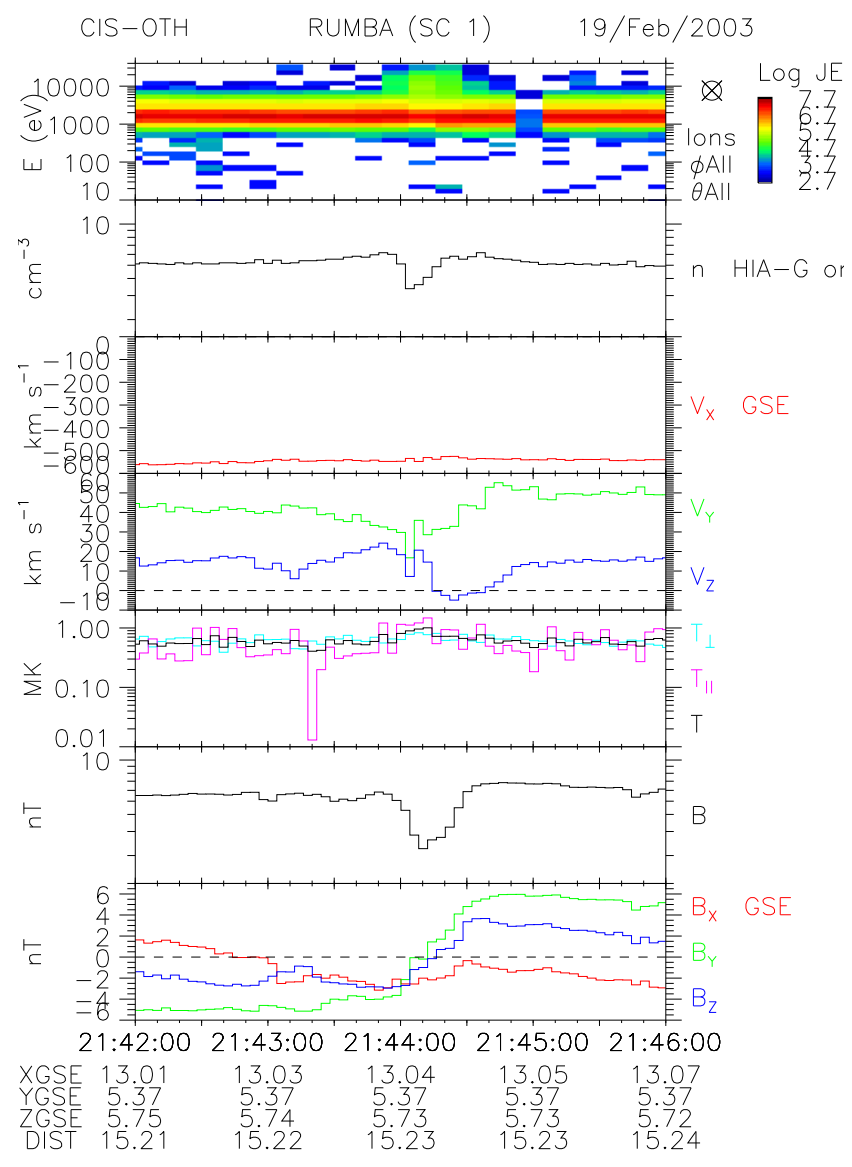

Fig. 8. 19 February 2003 21:42-21:46 UT summary plot. Same format as for Fig. 1.

reductions in the density hole depth as it is successively observed over time. The magnetic field intensities, in contrast, show no clear trend in depth. The difference may be accounted for by large-amplitude $7.5 \mathrm{~s}$ magnetic field waves occurring within the density hole, which when superposed on top of macroscopic structure could significantly modify instantaneous minima. The previous event showing density hole growth was recorded when the spacecraft were in a configuration that was elongated along $x_{\mathrm{GSE}}$, while in this case the formation was much closer to an equal-sided tetrahedron with separations of $\sim 4000 \mathrm{~km}$. We favor the interpretation that the $\phi_{\mathrm{SC}}$ values best reflect the temporal change to the structure, with the $7.5 \mathrm{~s}$ waves leaving the density unperturbed.

\subsection{February 2004, no-shear density hole}

For the final case example we present in Fig. 10 a density hole observed simultaneously with very coherent UFL waves, which are commonly associated with gyrating ion distributions in oblique foreshocks (Meziane et al., 2001; Mazelle et al., 2003). This is also of interest because it was

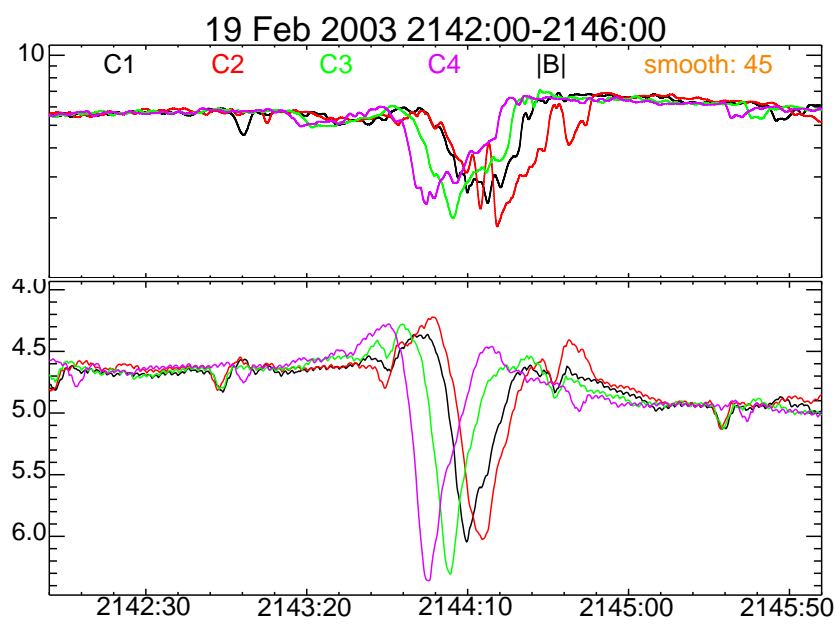

Fig. 9. 19 February 2003 21:42-21:46 UT, magnetic field intensity and $\phi_{\mathrm{SC}}$.

Table 1. Occurrence counts for embedding foreshock geometries.

\begin{tabular}{cccccc}
\hline$Q_{\perp}$ & oblique & $Q_{\|}$ & $Q_{\perp} \leftrightarrow Q_{\|}$ & $Q_{\perp} \leftrightarrow$ oblique & unknown \\
\hline 5 & 2 & 10 & 15 & 2 & 3 \\
\hline
\end{tabular}

one of only two events (out of 37) that lacked a clear crossstructure magnetic shear, with $\theta_{\Delta B}=7 \pm 10^{\circ}$. (The other event was embedded within a $Q_{\|}$foreshock.) As in the previous case the solar wind speed was $\sim 600 \mathrm{~km} \mathrm{~s}^{-1}$, and the flow deviation was minimal. (Here the perturbations to $v_{x}$ were larger, but still weak, at C1.)

\section{Statistical examination of density holes}

Using the selection criteria described in Sect. 2 we found $\sim 70$ "isolated" density hole events (without large adjacent upstream perturbations). Of these, 37 were suitable for characterization, with the remainder having problematic structures that could not cleanly be delimited. The latter included, e.g., structures with non-unique minima or indicators of successive density holes for which determinations of $\theta_{\Delta B}$ were impossible. The case studies presented above show various types of embedding foreshocks, and we have examined the particle and magnetic field signatures for all density holes in our sample to categorize them as well. These results are summarized in Table 1. The significant magnetic shears across some structures resulted in a boundary between two different types of foreshocks, in which case the embedding foreshocks were determined to be transitional, e.g. $Q_{\perp} \leftrightarrow$ oblique. While the $Q_{\|} \rightarrow Q_{\perp}$ transitions number 9 and the $Q_{\perp} \rightarrow Q_{\|}$transitions number 6 , the statistics do not permit us to conclude a significant preference for one sequence over another. Nor do we expect a physical basis for any difference, as the direction 
of the IMF rotation in combination with the location of the spacecraft determine the sequence. The statistics do allow us to see that density holes occur in a wide range of foreshock domains.

Characterization of other density hole attributes required a determination of interior and exterior plasma and field values. This was facilitated with an interactive routine that displayed the data and allowed the user to select representative upstream and downstream regions, and to delimit the extent of the density cavities. The final case example (Sect. 3.5 above) presented selection intervals (Fig. 10) for a near-worst case example, 29 February. In order to minimize aliasing effects, the exterior regions were selected to contain an integral number of the coherent pulsations seen, and the hole boundaries were selected to match where the interior densities fell below the mean values for the exterior intervals. The routine also drew horizontal fiducial lines corresponding to $2 \times$ the mean upstream values for $B$ and $n$, in order to help establish that the events were within the maximum upstream pulsation size. Average exterior plasma and field conditions, along with variances, were computed and saved in a database. For the results presented below, we take as the density hole interior values spin-resolution plasma and field measurements obtained at the instant of each hole's density minimum (all parameters subscripted with "min").

An important parameter is the angle between the embedded current sheet and the motional electric field. We have determined the current sheet normals using the coplanarity method due to Colburn and Sonett (1966). As a consistency check these values were compared with those obtained using the methods due to Abraham-Shrauner (1972) and AbrahamShrauner and Yun (1976), which yielded similar results. Below follow some of the more salient results.

\subsection{Occurrence statistics}

Figure 11 presents frequency histograms of various parameters determined for each of the density holes in the statistical sample. Black symbols and text correspond to $\mathrm{C} 1$, with green used for $\mathrm{C} 3$. Of the 37 events, there were 5 for which good determinations could not be made for $\mathrm{C} 3$, and 2 for which only C3 measurements were used. The first panel shows average upstream solar wind speeds, which were $638 \pm 105 \mathrm{~km} \mathrm{~s}^{-1}$ and $603 \pm 129 \mathrm{~km} \mathrm{~s}^{-1}$, for $\mathrm{C} 1$ and $\mathrm{C} 3$, respectively. (Mean values are denoted by $\mu$, standard deviations by $\epsilon$ and medians by $m$.) Differences between the two spacecraft are accounted for by the fact that of the 5 events included for C1 only, three had solar wind speeds $>700 \mathrm{~km} \mathrm{~s}^{-1}$, while the two events exclusive to $\mathrm{C} 3$ had solar wind speeds $\sim 325 \mathrm{~km} \mathrm{~s}^{-1}$.

The second (top right) panel shows event durations, which we regard as a rough proxy for scale size. However, the orientations found for the IMF current sheets were fairly uniformly distributed, suggesting that many $\Delta_{t}$ values should be lengthened by significant cosine factors as they transited the

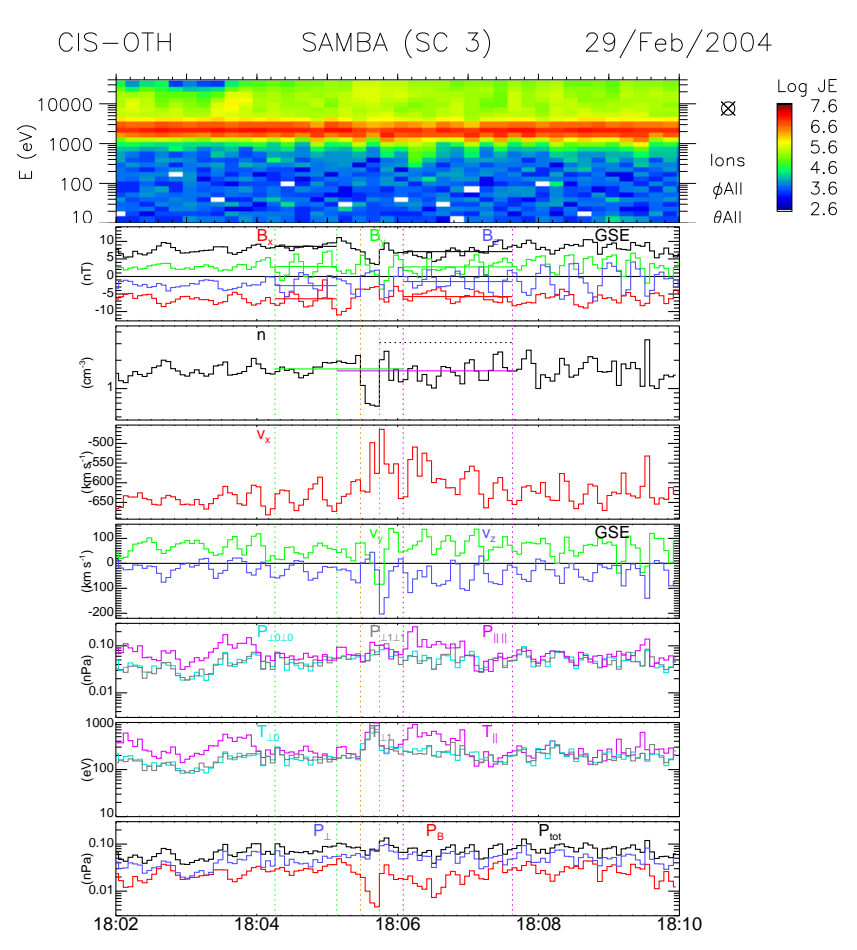

Fig. 10. 29 February 2004 18:02-18:10 UT summary plot. HIA ion energy spectrogram, $\boldsymbol{B}$, and various HIA moments. Representative selection intervals indicated (see text for details).

spacecraft. (No attempt has been made to correct for this.) The mean values of $32.0 \pm 20.0 \mathrm{~s}$ and $27.9 \pm 13.5 \mathrm{~s}$ for $\mathrm{C} 1$ and C3, respectively, are marginally larger than found in an earlier study (Parks et al., 2006) that was not constrained to "isolated" density holes.

The third panel shows a distribution of the cross-structure magnetic shear angle $\theta_{\Delta B}$, which largely reproduces the result of Parks et al. (2006), with moderately larger average shear values. The average values of $54.2 \pm 38.8^{\circ}$ and $52.7 \pm 39.0^{\circ}$, respectively, correspond to a preponderance of events with $\theta_{\triangle B}<90^{\circ}$. This contrasts with hot flow anomalies that tend to have substantially larger $\theta_{\Delta B}$ values. The fourth frame presents frequencies of the plasma flow deviation angles $\theta_{\Delta v}$, which are determined from the flows upstream and during measurements obtained at density minima. With mean flow perturbations near $4.8^{\circ}$, this sample of density holes has deviations much lower than seen for HFAs (for which large flow deviations are a defining characteristic).

The majority of events have $n_{\text {ext }} / n_{\min }<20$, with the mean values of $10.1 \pm 12.2$ and $7.7 \pm 8.1$ representing a substantial interior solar wind beam reduction in most cases. The distribution of temperature ratios are rather comparable, and likely have their origins in the same fact. As the cold solar wind beam is expelled from these structures the isotropic suprathermal components make a more significant contribution to the temperature moments. 

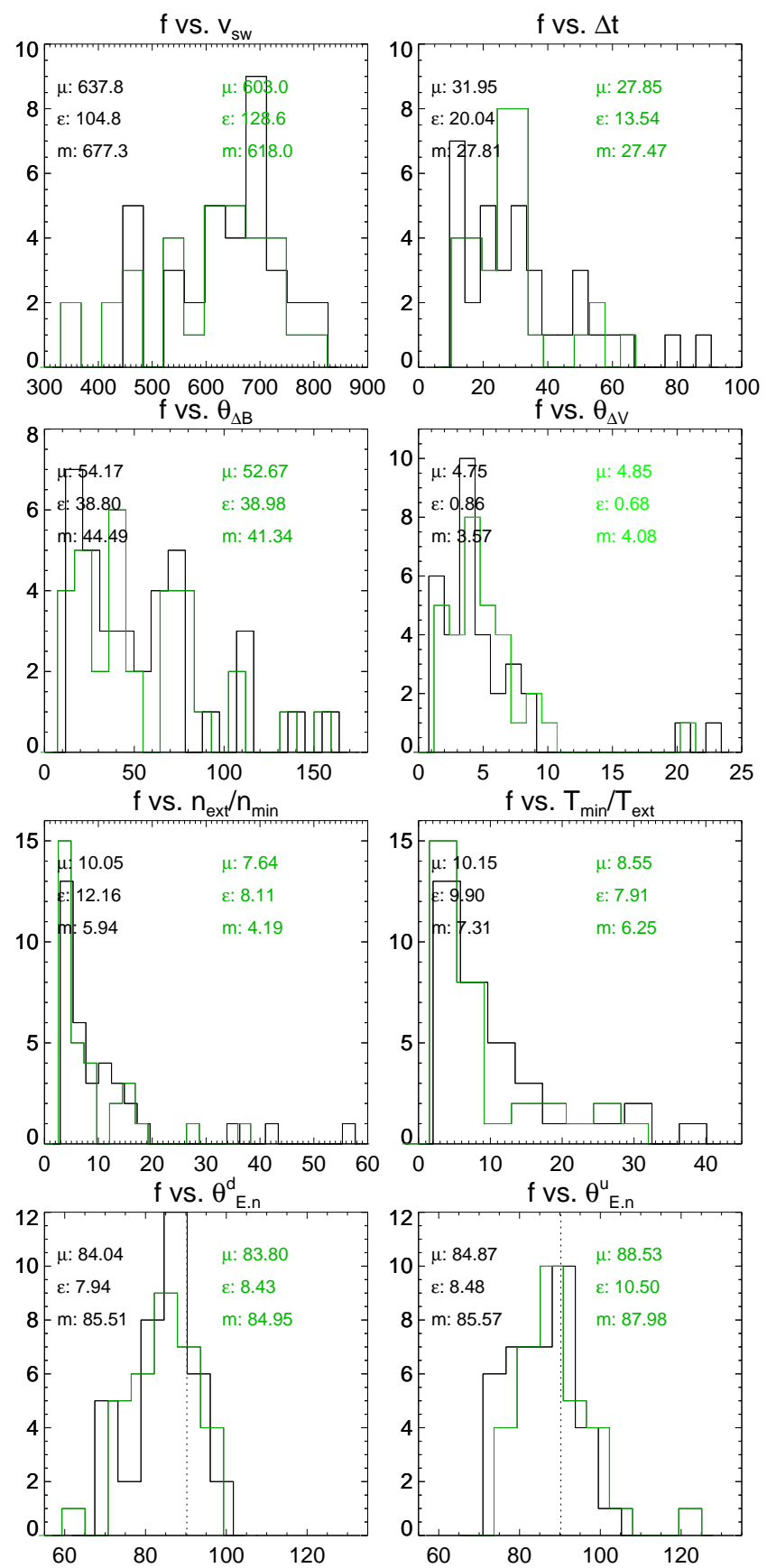

Fig. 11. Frequency distributions: $f$ vs. $v_{s w} ; f$ vs. $\Delta_{t} ; f$ vs. $\theta_{\Delta B}$; $f$ vs. $\theta_{\Delta v} ; f$ vs. $n_{\text {ext }} / n_{\min } ; f$ vs. $T_{\min } / T_{\text {ext }} ; f$ vs. $\theta_{\boldsymbol{E} \cdot \boldsymbol{n}}^{d} ; f$ vs. $\theta_{\boldsymbol{E} \cdot \boldsymbol{n}}^{u}$; $\mathrm{C} 1$ results are black; those for $\mathrm{C} 3$ are green.

The bottom two panels show distributions of the angles the convection electric fields $\boldsymbol{E}_{\text {conv }}$ make with the inferred current sheet normals for the downstream and upstream sides, respectively. These angles are defined such that values $<90^{\circ}$ correspond to electric fields pointing outward. The downstream angles $\theta_{\boldsymbol{E} \cdot \boldsymbol{n}}^{d}$ have mean values that are lower than $90^{\circ}, 84.0 \pm 7.9^{\circ}$ and $83.8 \pm 8.4^{\circ}$ for $\mathrm{C} 1$ and $\mathrm{C} 3$, respectively. Again, this contrasts with the determinations made for HFAs
(Schwartz et al., 2000; Paschmann et al., 1988; Thomsen et al., 1993), which have the normal components to the fields pointing inward. The upstream angles $\theta_{\boldsymbol{E} \cdot \boldsymbol{n}}^{u}$ are $84.9 \pm 8.5^{\circ}$ and $88.5 \pm 10.5^{\circ}$. The statistics for $\mathrm{C} 3$ are not compelling, although removal of the outlier at $125^{\circ}$ would shift the peak another $1.5^{\circ}$ from $90^{\circ}$.

\subsection{Correlations}

We have tested for correlations between several parameters $-\Delta_{t}, \theta_{\Delta v}, \theta_{\Delta B}, v_{s w}, n_{\mathrm{ext}} / n_{\min }, T_{\min } / T_{\mathrm{ext}}, \theta_{\boldsymbol{E} \cdot \boldsymbol{n}}^{u}$ and $\theta_{\boldsymbol{E} \cdot \boldsymbol{n}}^{d}-$ to look for indications of controlling factors in the development of density holes. For the modest number of events in the sample, only a handful of relationships were manifest, which we present in Fig. 12. The most striking relationship found is between $T_{\min } / T_{\text {ext }}$ and $n_{\text {ext }} / n_{\text {min }}$, which we have accounted for above as resulting from interior temperatures being increasingly dominated by the supra-thermal population as the cold, dense solar wind beam is excluded. The only dependence upon $v_{s w}$ - and a weak one at that - is for $n_{\text {ext }} / n_{\min }$. This may be related to the high average solar wind speeds observed for this sample; if decreases in $v_{s w}$ lead to weaker density holes, they will be increasingly difficult to detect.

The parameters most inclined to bear out correlations with other parameters are the angles between $\boldsymbol{E}_{\text {conv }}$ and the downstream and upstream IMF current sheet normals, $\theta_{\boldsymbol{E} \cdot \boldsymbol{n}}^{d}$ and $\theta_{\boldsymbol{E} \cdot \boldsymbol{n}}^{u}$, respectively. The third panel of Fig. 12 shows a weak, but probable relationship between $\theta_{\boldsymbol{E} \cdot \boldsymbol{n}}^{d}$ and $\Delta_{t}(p<0.04$ and $p<0.1$ for $\mathrm{C} 1$ and $\mathrm{C} 3$ ). It is notable that the outwardpointing normal component approaches $0\left(\theta_{\boldsymbol{E} \cdot \boldsymbol{n}}^{d} \rightarrow 90^{\circ}\right)$ as $\Delta_{t}$ increases; larger events appear to have weaker normal electric field components. This dependence, while suggested by the best fit line, is not statistically demonstrated for the upstream side of the structure, however (fourth panel). More compelling are the relationships between $\theta_{\boldsymbol{E} \cdot \boldsymbol{n}}$ and $\theta_{\Delta B}$ (bottom panels). As $\theta_{\Delta B} \rightarrow 180^{\circ}, \theta_{\boldsymbol{E} \cdot \boldsymbol{n}}$ decreases from $90^{\circ}$ (tangency) to $75-80^{\circ}$. This is to say that as the cross-structure magnetic shear increases, the normal component of the motional electric field is increasingly outward.

\section{Discussion}

We began Sect. 3 by presenting a hot flow anomaly studied by Lucek et al. (2004b). In addition to its relatively long duration of $\sim 5.5 \mathrm{~min}$, this was characterized by very large flow deviations, broad magnetosheath-like ion distributions, and magnetic field intensities that were usually, but not always larger than the solar wind values. We noted a density hole-like structure on the upstream edge, with a brief correlated field and density depression, and a momentary increase in temperature. Other instances of such edge structures exist, such as for an HFA observed near 04:16 UT on 2 April 2002 (shortly after the density hole presented in Sect. 3.2, not shown), which had a density hole-like structure at its 
downstream limit. It is difficult to draw conclusions, given that the highly-structured magnetic fields observed are local to the spacecraft, and may not reflect global conditions, but possibilities include HFA expansion into regions containing density holes, HFAs being built up out of merging density holes, and the emergence of density holes out of embedded turbulence that may produce conditions favorable for density hole creation. Based upon the statistical results of Sect. 4, we conclude (below) that density holes have a different genesis and are unlikely to form HFAs through merging.

In Sect. 3.2 we presented what we consider to be an archetypal "isolated" density hole, which merits detailed discussion. It is centered upon an IMF tangential discontinuity, exhibits a fast mode-like character on time scales corresponding to spatial extents $\gtrsim 2000 \mathrm{~km}$ in the structure frame, has an order of magnitude temperature excess over surrounding solar wind, and moderate flow deviations. The ion distributions have two populations (Fig. 5), with an isotropic supra-thermal population and a solar wind beam intensity that has been greatly reduced. These characteristics are typical of density holes overall. Differences between the higherresolution magnetic field profiles and the proxy densities provided by spacecraft potentials suggest that the fast mode-like character breaks down on scales corresponding to $\lesssim 500 \mathrm{~km}$. This is not a surprise, as these are of the order of a solar wind ion Larmor radius, for which a break-down of MHD behavior might be expected.

We noted an asymmetry in the structure profile, with a dispersive decrease in the solar wind beam flux on the downstream side. We have found one event that shows a reverse asymmetry, indicating that the affect can occur on both the upstream and downstream edges. (Other events show "reverse" asymmetry in the density profiles, but were not observed in burst mode, for which the spectrograms could resolve the dispersion.) If some mechanism prevents new, thermal solar wind ions from gaining access to a density hole field line, more-rapid streaming along these flux tubes may lead to losses of the most energetic ions first, with the deepest part of the hole being the location where such a loss process may have been in effect the longest. This picture is not consistent, however, with rarefaction due to explosive expansion caused by an interior overpressure. Testing for excess interior pressure is difficult, if not impossible, since the RAPID solid state detectors on Cluster do not provide measurements with high enough sampling rates, while a substantial partial pressure may be present above $40 \mathrm{keV}$. Efforts to analyze the stress balance at density hole edges have so far yielded no conclusive results.

The magnetic structure of the 2 April 2002 density hole is interesting as the variance and multi-point timing analysis points to a rotational discontinuity on the upstream edge embedded within a larger tangential discontinuity across the whole structure. Although RDs are consistent with reconnection topologies, it remains to be seen whether tearing could take place in the presence of backstreaming ions that have
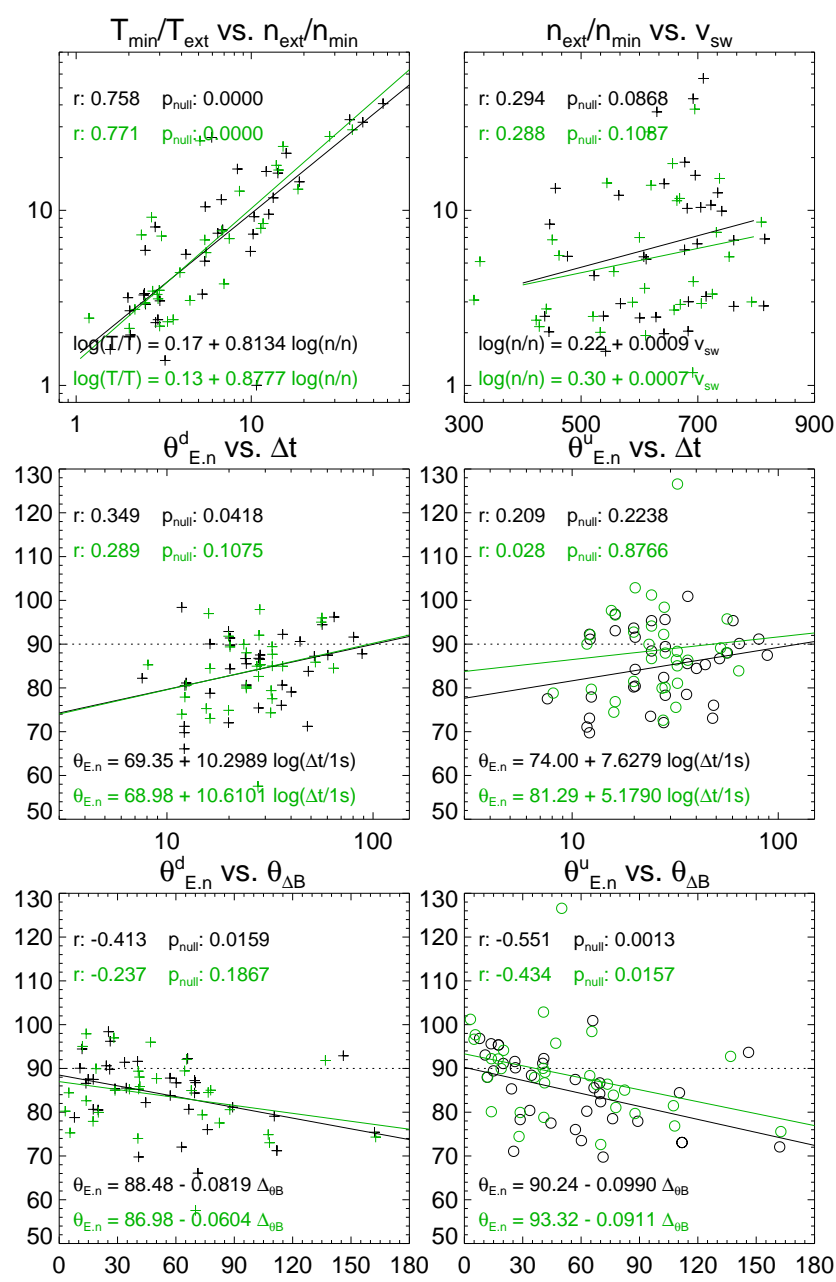

Fig. 12. Correlations. $T_{\min } / T_{\text {ext }}$ vs. $n_{\text {ext }} / n_{\min } ; n_{\text {ext }} / n_{\min }$ vs. $v_{s} w$; $\theta_{\boldsymbol{E} \cdot \boldsymbol{n}}^{d}$ vs. $n_{\mathrm{ext}} / n_{\min } ; \theta_{\boldsymbol{E} \cdot \boldsymbol{n}}^{u}$ vs. $n_{\mathrm{ext}} / n_{\mathrm{min}} ; \theta_{\boldsymbol{E} \cdot \boldsymbol{n}}^{d}$ vs. $\Delta_{t} ; \theta_{\boldsymbol{E} \cdot \boldsymbol{n}}^{u}$ vs. $\Delta_{t} ;$ $\theta_{\boldsymbol{E} \cdot \boldsymbol{n}}^{d}$ vs. $\theta_{\Delta B} ; \theta_{\boldsymbol{E} \cdot \boldsymbol{n}}^{u}$ vs. $\theta_{\Delta B}$.

plasma frame parallel speeds many times in excess of the Alfvén speed. The RD topology is interesting as it would suggest easy streaming access between the density hole interior and the upstream. This seems to be contradicted by the remote-sensing distribution for supra-thermal ions (Fig. 5) that indicates strict confinement to guiding centers within the density hole, and by the abrupt drop in thermal solar wind ions at that edge.

The timing analysis on the upstream edge of this structure indicates that it is standing relative to the flow at approximately the upstream Alfvén speed. The 0.35 fractional uncertainty allows for values ranging from sub-Alfvénic to super-fast mode values, although a supercritical relative speed is unlikely. This relatively deep density hole with $n_{\text {ext }} / n_{\min } \sim 20$, indicates substantial changes from the ambient solar wind medium by this stage of development. The local maxima in $B$ and $\phi_{\mathrm{SC}}$ at 04:12:41 UT may indicate the beginning of an upstream overshoot, and an intriguing 
possibility is that the density hole formation is an antecedent to subsequent upstream pulsation growth, rather than the reverse as was proposed by Behlke et al. (2003).

A few cases, such as the event shown in Sect. 3.3, indicate substantial growth (increasing density depletion) of density holes during the 15-30 s spanning first and last observations by Cluster spacecraft. This corroborates a case event presented by Parks et al. (2006), which was observed first by Cluster, and then by Double Star. Our third density hole case event (Sect. 3.4) suggests instead a measurable decay over $\sim 15 \mathrm{~s}$, which raises the prospect of these events being rather transient. Whether forming or decaying, rapid temporal variation is certain to muddy the statistical results of Sect. 4. As a proxy for size, $\Delta_{t}$ is subject to projection effects due to variable structure orientations, as well as to the stage of development at which an event is observed.

In combination, the different case events presented, and the summary of embedding foreshock geometries for the statistical sample (Table 1), show that density holes can occur over a wide range of foreshock geometries. We therefore conclude that geometry does not significantly constrain their formation, nor does the presence of differing backstreaming ion populations or ULF waves. While HFA phenomenology appears to be strongly dependent upon the magnetic shear of TDs interacting with the bow shock, it is notable that most of the density holes in this study have $\theta_{\Delta B}$ values $<44^{\circ}$. The only discernible relationship with $\theta_{\Delta B}$ involved $\theta_{\boldsymbol{E} \cdot \boldsymbol{n}}$. The tendency for the normal component of $\boldsymbol{E}_{\text {conv }}$ to point increasingly away from the structure as $\theta_{\Delta B}$ increases further distinguishes these structures from HFAs. However, the absence of a dependence for physical characteristics such as $T_{\min } / T_{\text {ext }}$ or $\theta_{\Delta v}$ might be masked by the varying phases of development at which events are captured. Hybrid simulations by Thomsen et al. (1993) predict much weaker interactions between tangential discontinuities and shocks when $\boldsymbol{E}_{\text {conv }}$ has components pointing outward on both sides. These predict no edge enhancements, and notably do not produce density cavities. It may be the case that charge separation effects are important, which would require full particle methods. The flow deviations reported here $\left(\theta_{\Delta v} \sim 5^{\circ}\right)$ are much smaller than previously indicated, and the difference may be accounted for by our selection of "isolated" events; large upstream pulsations are likely to divert plasma flows.

It is notable that these events are found to occur for relatively high solar wind speeds $\sim 620 \mathrm{~km} \mathrm{~s}^{-1}$. The high $v_{s w}$ values may in fact suffer from selection bias, as we have restricted ourselves to measurements obtained with the magnetosheath mode of the HIA detector. In other words, these observations were constrained by mode settings that were designed to capture the foreshock when it was at or inside of the nominal shock location. This would predispose the events to occur during higher than average dynamic pressure intervals. (Had we selected events for which the analyzer was in solar wind mode, our sample would have been similarly biased towards lower than average dynamic pressure intervals; mixing both data sets, having different sources of measurement error, would have also introduced complications.) We note, however, that the frequency histogram for $v_{s w}$ (Fig. 11) is two-tailed, indicating that it is not seriously constrained at its lower limit. S̆afránková et al. (2000) had found solar wind speeds nearly as high in their survey of magnetosheath HFAs.

A final consideration is that the distribution of IMF current sheet orientations we found (not shown) were fairly uniformly distributed in cone angle $\theta_{v n}$. Large cone angles favor slow current sheet motions along the shock surface near the subsolar point. Burgess (1989); Schwartz et al. (2000) have argued that low contact speeds would be favorable for injecting shock-reflected ions into the current sheet.

\section{Conclusions}

We have presented case examples of foreshock density holes, and survey results from a sample of 37 events. In order to minimize the influence of upstream structures that could significantly alter plasma conditions, we have specifically selected events that lack strong trailing pulsations and density overshoots. Density holes can be observed to develop significantly over time scales as short as $\sim 10 \mathrm{~s}$, and may have comparably short lifetimes. The dispersive signatures seen in the few burst mode ion spectrograms may indicate that density holes do not form through explosive expansion following strong heating. In addition to confirming results obtained using broader selection criteria, the simple existence of a statistical sample of such isolated density holes suggests that large-amplitude pulsations are not required for their formation.

As is the case for HFAs, density holes are observed over a wide range of foreshock geometries, are preferentially seen during fast solar wind flows $\left(v_{s w} \sim 620 \mathrm{~km} \mathrm{~s}^{-1}\right)$, and are embedded within IMF current sheets. Unlike HFAs, isolated density holes lack particles with energies below that of the solar wind, and flow deviations tend to be rather small. (The large flow deviations for previously-identified events can be accounted for by large upstream pulsations, which stand relative to the solar wind flow with large normal incidence speeds and likely cause the deflections in a manner similar to fast mode shocks.) Additional characteristics that further distinguish density holes from hot flow anomalies include magnetic shears that are typically much lower $\left(\left\langle\theta_{\Delta B}\right\rangle \sim 50-55^{\circ}\right)$, and normal components for the motional electric field that tend to point outward from, rather than into, the current sheets on both the downstream and upstream sides. This inverted field scenario had been modeled (Thomsen et al., 1993), but density holes were not predicted. The similarities in characteristics for the particular hot diamagnetic cavities identified by Thomsen et al. (1986) suggest merely a difference in degree or development, but their identification with 
inward-pointing motional electric field components seems to make these HDCs fundamentally distinct as well.

Finally, density holes seem to be unrelated to the foreshock cavities described by, e.g. Sibeck et al. (2001), or to long-studied solar wind magnetic holes (Turner et al., 1977). Foreshock cavities appear to occur where $Q_{\|}$foreshock domains are embedded within $Q_{\perp}$ regions, while density holes are found to be embedded within either type, or combination, of foreshock domains. Magnetic holes exhibit field intensity depletions, but not the defining, correlated density drops of density holes. The apparent association of the latter with the ion foreshock points to a mechanism involving backstreaming ions or the bow shock itself, while magnetic holes are seen throughout the heliosphere, where no shock association is manifest.

Acknowledgements. M. Wilber would like to thank E. Lucek and A. Hull for helpful discussions. This work was supported by NASA grants NNG05GF99G and NASA-NNX07AP96G. We gratefully acknowledge the support of the FGM and EFW teams for magnetic fields and spacecraft potential data, and the Cluster Active Archive.

Topical Editor R. Nakamura thanks M. Goldstein and another anonymous referee for their help in evaluating this paper.

\section{References}

Abraham-Shrauner, B.: Determination of magnetohydrodynamic shock normals, J. Geophys. Res., 77, 736-739, 1972.

Abraham-Shrauner, B. and Yun, S.: Interplanetary shocks seen by Ames plasma probe on Pioneer 6 and 7, J. Geophys. Res., 81, 2097-2102, 1976.

Anderson, K. A.: Measurements of Bow Shock Particles Far Upstream From the Earth, J. Geophys. Res., 86, 4445-4454, 1981.

Balogh, A., Carr, C. M., Acũna, M. H., Dunlop, M. W., Beek, T. J., Brown, P., Fornacon, K.-H., Georgescu, E., Glassmeier, K.-H., Harris, J., Musmann, G., Oddy, T., and Schwingenschuh, K.: The cluster Magnetic field investigation: overview of in-flight performance and initial results, Ann. Geophys., 19, 1207-1217, 2001, http://www.ann-geophys.net/19/1207/2001/.

Baumgärtel, K.: Soliton approach to magnetic holes, J. Geophys. Res., 104, 28 295-28 308, 1999.

Behlke, R., André, M., Buchert, S., Vaivads, A., and Eriksson, A.: Multi-point electric field measurements of Short LargeAmplitude Magnetic Structures (SLAMS) at the Earth's quasiparallel bow shock, Geophys. Res. Lett., 30, 1177, doi:10.1029/ 2002GL015871, 2003.

Bonifazi, C. and Moreno, G.: Reflected and Diffuse Ions Backstreaming from the Earth's Bow Shock 1, Basic Properties, J. Geophys. Res., 86, 4381-4396, 1981a.

Bonifazi, C. and Moreno, G.: Reflected and Diffuse Ions Backstreaming from the Earth's Bow Shock 2, Origin, J. Geophys. Res., 86, 4397-4404, 1981b.

Burgess, D.: On the effect of a tangential discontinuity on ions specularly reflected at an oblique shock, J. Geophys. Res., 94, 472478, 1989.

Burlaga, L. and Lemaire, J.: Interplanetary Magnetic Holes: Theory, J. Geophys. Res., 83, 5157-5160, 1978.
Buti, B., Tsurutani, B., Neugebauer, M., and Goldstein, B.: Generation Mechanism for Magnetic Holes in the Solar Wind, Geophys. Res. Lett., 28, 1355-1358, 2001.

Colburn, D. and Sonett, C.: Discontinuities in the solar win, Space Sci. Rev., 5, 439-506, 1966.

Eastwood, J., Sibeck, D., Angelopoulos, V., Phan, T., Bale, S., McFadden, J., Cully, C., Mende, S., Larson, D., Frey, S., Carlson, C., Glassmeier, K.-H., Auster, H., Roux, A., and Le Contel, O.: THEMIS observations of a hot flow anomaly: Solar wind, magnetosheath, and ground-based measurements, Geophys. Res. Lett., 35, L17S03, doi:10.1029/2008GL033475, 2008.

Fitzenreiter, R. and Burlaga, L.: Structure of Current Sheets in Magnetic Holes at 1 AU, J. Geophys. Res., 83, 5579-5585, 1978.

Fuselier, S., Thomsen, M., Gosling, J., Bame, S., and Russell, C.: Fast shocks at the edges of hot diamagnetic cavities upstream from the earth's bow shock, J. Geophys. Res., 92, 3187-3194, 1987.

Fuselier, S. A., Thomsen, M. F., Gosling, J. T., Bame, S. J., and Russell, C. T.: Gyrating and intermediate ion distributions upstream from the Earth's bow shock, J. Geophys. Res., 91, 91-99, 1986.

Greenstadt, E. W., Green, I. M., Inouye, G. T., Colburn, D. S., Binsack, J. H., and Lyon, E. F.: Dual satellite observations of Earth's bow shock I: The thick pulsation shock, Cosmic Electrodyn., 1, 160-177, 1970a.

Greenstadt, E. W., Green, I. M., Inouye, G. T., Colburn, D. S., Binsack, J. H., and Lyon, E. F.: Dual satellite observations of Earth's bow shock II: Field aligned upstream waves, Cosmic Electrodyn., 1, 279-296, 1970b.

Greenstadt, E. W., Scarf, F. L., Russell, C. T., Holzer, R. E., Formisano, V., Hedgecock, P. C., and Neugebauer, M.: Structure of a quasi-parallel, quasi-laminar bow shock, J. Geophys. Res., 82, 651-666, 1977.

Gustafsson, G., Boström, R., Holback, B., Holmgren, G., Lundgren, A., Stasiewicz, K., Åhlén, L., Mozer, F., Pankow, D., Harvey, P., Berg, P., Ulrich, R., Pedersen, A., Schmidt, R., Butler, A., Fransen, A., Klinge, D., Thomsen, M., Fälthammar, C.-G., Lindqvist, P.-A., Christenson, S., Holtet, J., Lybekk, B., Sten, T., Tanskanen, P., Lappalainen, K., and Wygant, J.: The electricfield and wave experiment for the Cluster mission, Space Sci. Rev., 79, 137-156, 1997.

Harvey, C. C.: Analysis Methods for Multi-Spacecraft Data, chap. Spatial Gradients and the Volumetric Tensor, pp. 307-322, ISSI Scientific Report, ESA Publications Division, Keplerlaan 1, 2200 AG Noordwijk, The Netherlands, 1998.

Hoppe, M. M., Russell, C. T., Frank, L. A., Eastman, T. E., and Greenstadt, E. W.: Upstream hydromagnetic waves and their association with backstreaming ion populations - ISEE 1 and 2 observations, J. Geophys. Res., 86, 4471-4492, 1981.

Lin, N., Lee, E., Mozer, F., Parks, G. K., Wilber, M., and Rème, H.: Nonlinear low-frequency wave aspect of foreshock density holes, Ann. Geophys., 26, 3707-3718, 2008, http://www.ann-geophys.net/26/3707/2008/.

Lucek, E., Horbury, T., Balogh, A., Dandouras, I., and Rème, H.: Cluster obervations of structures at quasi-parallel bow shocks, Ann. Geophys., 22, 2309-2314, 2004a, http://www.ann-geophys.net/22/2309/2004/.

Lucek, E., Horbury, T., Balogh, A., Dandouras, I., and Rème, H.: Cluster obervations of hot flow anomalies, J. Geophys. Res., 109, 
A06207, doi:10.1029/2003JA010016, 2004b.

Lucek, E., Horbury, T., Dandouras, I., and Rème, H.: Cluster observations of the Earth's quasi-parallel bow shock, J. Geophys. Res., 113, A07S02, doi:10.1029/2007JA012756, 2008.

Lucek, E. A., Horbury, T. S., Dunlop, M. W., Cargill, P. J., Schwartz, S. J., Balogh, A., Brown, P., Carr, C., Fornacon, K.H., and Georgescu, E.: Cluster magnetic field observations at a quasi-parallel bow shock, Ann. Geophys., 20, 1699-1710, 2002, http://www.ann-geophys.net/20/1699/2002/.

Mann, G., Lühr, H., and Baumjohann, W.: Stastical analysis of short large-amplitude magnetic field structures in the vicinity of the quasi-parallel bow shock, J. Geophys. Res., 99, 13 315-13 323, 1994.

Mazelle, C., Meziane, K., Le Quéue, D., Wilber, M., Eastwood, J. P., Rème, H., Sauvaud, J.-A., Bosqued, J.-M., Dandouras, I., McCarthy, M., Kistler, L. M., Klecker, B., Korth, A., BavassanoCattaneo, M. B., Lundin, R., and Balogh, A.: Production of Gyrating Ions from Nonlinear Wave-Particle Interaction Upstream from the Earth's Bow Shock: a Case Study from Cluster-CIS, Planet. Space Sci., 51, 785-795, 2003.

Meziane, K., Lin, R. P., Parks, G. K., Larson, D. E., Bale, S. D., Mason, G. M., Dwyer, J. R., and Lepping, R. P.: Evidence for acceleration of ions to $\sim 1 \mathrm{MeV}$ by adiabatic-like reflection at the quasi-perpendicular Earth's bow shock, Geophys. Res. Lett., 26, 2925-2928, 1999.

Meziane, K., Mazelle, C., Lin, R. P., LeQuéau, D., Larson, D. E., Parks, G. K., and Lepping, R. P.: Three-dimensional observations of gyrating ion distributions far upstream from the Earth's bow shock and their associated with low-frequency waves, J. Geophys. Res., 106, 5731-5742, 2001.

Meziane, K., Wilber, M., Lin, R. P., and Parks, G. K.: Gyrophase-Restricted $100 \mathrm{keV}-2 \mathrm{MeV}$ Ion Beams Near the Foreshock Boundary, Geophys. Res. Lett., 30, 2049, doi:10. 1029/2003GL017592, 2003.

Meziane, K., Wilber, M., Mazelle, C., Parks, G. K., and Hamza, A. M.: A review of field-aligned beams observed upstream of the bow shock, in: The Physics of Collisionless Shocks, 4th Annual IGPP International Astrophysics Conference, edited by: Li, G., Zank, G. P., and Russell, C. T., vol. 781, pp. 116-122, American Institute of Physics, 2005.

Parks, G., Lee, E., Mozer, F., Wilber, M., Lucek, E., Dandouras, I., Rème, H., Mazelle, C., Cao, J., Meziane, K., Goldstein, M., and Escoubet, P.: Larmor radius size density holes discovered in the solar wind upstream of Earth's bow shock, Phys. Plasmas, 13, 050701, doi:10.1063/1.2201056, 2006.

Parks, G., Lee, E., Lin, N., Mozer, F., Wilber, M., Lucek, E., Dandouras, I., Rème, H., Cao, J., Canu, P., Cornilleau-Wehrlin, N., Décréau, Goldstein, M., and Escoubet, P.: Density holes in the upstream solar wind, in: Turbulence and Nonlinear Processes in Astrophysical Plasmas; 6th Annual International Astrophysics Conference, edited by: Shaikh, D. and Zank, G., vol. 932 of AIP Conference Proceedings, pp. 9-15, American Institute of Physics, doi:10.1063/1.2778939, 2007.

Paschmann, G., Sckopke, N., Papamastorakis, I., Asbridge, J. R., Bame, S. J., and Gosling, J. T.: Characteristics of Reflected and Diffuse Ions Upstream from the Earth's Bow Shock, J. Geophys. Res., 86, 4355-4364, 1981.

Paschmann, G., Haerendel, G., Sckopke, N., Möbius, E., Lühr, H., and Carlson, C.: Three-dimensional plasma structures with anomalous flow directions near the Earth's bow shock, J. Geophys. Res., 93, 11 279-11294, 1988.

Rème, H., Aoustin, C., Bosqued, J. M., Dandouras, I., Lavraud, B., Sauvaud, J. A., Barthe, A., Bouyssou, J., Camus, T., Coeur-Joly, O., Cros, A., Cuvilo, J., Ducay, F., Garbarowitz, Y., Medale, J. L., Penou, E., Perrier, H., Romefort, D., Rouzaud, J., Vallat, C., Alcayde, D., Jacquey, C., Mazelle, C., d'Uston, C., Möbius, E., Kistler, L. M., Crocker, K., Granoff, M., Mouikis, C., Popecki, M., Vosbury, M., Klecker, B., Hovestadt, D., Kucharek, H., Kuenneth, E., Paschmann, G., Scholer, M., Sckopke†, N., Seidenschwang, E., Carlson, C. W., Curtis, D. W., Ingraham, C., Lin, R. P., McFadden, J. P., Parks, G. K., Phan, T., Formisano, V., Amata, E., Bavassano-Cattaneo, M. B. Baldetti, P., Bruno, R., Chionchio, G., Di Lellis, A., Marcucci, M. F., Pallocchia, G., Korth, A., Daly, P. W., Graeve, B., Rosenbauer, H., Vasyliunas, V., McCarthy, M., Wilber, M., Eliasson, L., Lundin, R., Olsen, S., Shelley, E. G., Fuselier, S., Ghielmetti, A. G., Lennartsson, W., Escoubet, C. P., Balsiger, H., Friedel, R., Cao, J.-B., Kovrazhkin, R. A., Papamastorakis, I., Pellat, R., Scudder, J., and Sonnerup, B. U. Ö.: First multispacecraft ion measurements in and near the Earth's magnetosphere with identical Cluster ion spectrometry (CIS) experiment, Ann. Geophys., 19, 1303-1354, 2001, http://www.ann-geophys.net/19/1303/2001/.

Russell, C. and Greenstadt, E.: Initial ISEE magnetometer results: Shock observations, Space Sci. Rev., 23, 3-37, 1979.

S̆afránková, J., Přech, L., Nĕmeček, Z., Sibeck, D., and Mukai, T.: Magnetosheath response to the interplanetary magnetic field discontinuity, J. Geophys. Res., 105, 25 113-25 121, 2000.

S̆afránková, J., Přech, L., Nĕmeček, Z., and Sibeck, D.: The structure of hot flow anomalies in the magnetosheath, Adv. Space Res., 30, 2737-2744, 2002.

Sarris, E. T., Krimigis, S. M., , and Armstrong, T. P.: Observations of a High Energy Shock Spike in Interplanetary Space, Geophys. Res. Lett., 3, 133-136, 1976.

Schwartz, S., Chaloner, C., Christiansen, P. J., Coates, A., Hall, D., Johnstones, A., Gough, M., Norris, A., Rijnbeek, R. P., Southwood, D., and Woolliscroft, L.: An Active Current Sheet in the Solar Wind, Nature, 318, 269-271, 1985.

Schwartz, S., Sibeck, D., Wilber, M., Meziane, K., and Horbury, T.: Kinetic Aspects of Foreshock Cavities, Geophys. Res. Lett., 33, L12103, doi:10.1029/2005GL025612, 2006.

Schwartz, S. J.: Magnetic field structures and related phenomena at quasi-parallel shocks, Adv. Space Res., 11, (9)231-(9)240, 1991.

Schwartz, S. J. and Burgess, D.: Quasi-parallel shocks - A patchwork of three-dimensional structures, Geophys. Res. Lett., 18, 373-376, 1991.

Schwartz, S. J., Kessel, R. L., Brown, C. C., Woolliscroft, L. J. C., and Dunlop, M. W.: Active current sheets near the Earth's bow shock, J. Geophys. Res., 93, 11 295-11310, 1988.

Schwartz, S. J., Paschmann, G., Sckopke, N., Bauer, T. M., Dunlop, M., Fazakerley, A. N., and Thomsen, M. F.: Conditions for the formation of hot flow anomalies at Earth's bow shock, J. Geophys. Res., 105, 12 639-12650, doi:10.1029/1999JA000320, 2000.

Sentman, D. D., Kennel, C. F., and Frank, L. A.: Plasma Rest Frame Distributions of Suprathermal Ions in the Earth's Foreshock Region, J. Geophys. Res., 86, 150-152, 1981.

Sibeck, D., Borodkova, N., Schwartz, S., Owen, C., Kessel, R., Kokubun, S., Lepping, R., Lin, R., Liou, K., Lühr, H., McEntire, 
R., Meng, C.-I., Mukai, T., Nĕmeček, Z., Parks, G., Phan, T., Romanov, S., S̆afránková, J., Sauvaud, J.-A. Singer, H., Solovyev, S., Szabo, A. Takahashi, K., Williams, D., Yumoto, K., and Zastenker, G.: Comprehensive study of the magnetospheric response to a hot flow anomaly, J. Geophys. Res., 104, 4577-4593, 1999.

Sibeck, D., Decker, R., Mitchell, D., Lazarus, A., Lepping, R., and Szabo, A.: Solar wind preconditioning in the flank foreshock: IMP 8 observations, J. Geophys. Res., 106, 21 675-21 688, 2001.

Sibeck, D., Phan, T.-D., Lin, R., Lepping, R., and Szabo, A.: Wind obserations of foreshock cavities: A case Study, J. Geophys. Res., 107, 1271, doi:10.1029/2003JA010374, 2002.

Stevens, M. and Kasper, J.: A scale-free analysis of magnetic holes at $1 \mathrm{AU}$, J. Geophys. Res., 112, A05109, doi:10.1029/ 2006JA012116, 2007.

Thomas, V., Winske, D., Thomsen, M., and Onsager, T.: Hybrid simulation of the formation of a hot flow anomaly, J. Geophys. Res., 96, 11 625-11 632, 1991.

Thomsen, M., Gosling, J., Bame, S., Quest, K., and Russell, C.: On the origin of hot diamagnetic cavities near the Earth's bow shock, J. Geophys. Res., 93, 11311-11 325, 1988.

Thomsen, M., Gosling, J., Bame, S., and Russell, C.: Magnetic Pulsations at the Quasi-Parallel Shock, J. Geophys. Res., 95, 957966, 1990.
Thomsen, M., Thomas, V., Winske, J., and Gosling, J.: Observational test of hot flow anomaly formation by the interaction of a magnetic discontinuity with the bow shock, J. Geophys. Res., 98, 15 319-15330, 1993.

Thomsen, M. F., Gosling, J. T., Fuselier, S., Bame, S. J., and Russell, C. T.: Hot, diamagnetic cavities near the Earth's bow shock, J. Geophys. Res., 91, 2961-2973, 1986.

Tsurutani, B., Dasgupta, B., Galvan, C., Neugebauer, M., Lakhina, G., Arballo, J., Winterhalter, D., Goldstein, B., and Buti, B.: Phase-steepened Alfvén waves, proton perpendicular energization and the creation of magnetic holes and magnetic decreases: The ponderomotive force, Geophys. Res. Lett., 29, 2233, doi: 10.1029/2002GL015652, 2002.

Turner, J., Burlaga, L., Ness, N., and Lemaire, J.: Magnetic Holes in the Solar Wind, J. Geophys. Res., 82, 1921-1924, 1977.

Winterhalter, D., Neugebauer, M., Goldstein, B., Smith, E., Bame, S., and Balogh, A.: Ulysses field and plasma observations of magnetic holes in the solar wind and their relation to mirrormode structures, J. Geophys. Res., 99, 23 371-23 381, 1994.

Woolliscroft, L., Brown, C., Schwartz, S., Chaloner, C., and Christiansen, P.: AMPTE-UKS observations of current sheets in the solar wind, Adv. Space Res., 6, 89-92, doi:10.1016/ 0273-1177(86)90017-7, 1986. 